\title{
Evolution of predator foraging in response to prey infection favors species coexistence
}

\section{Loïc Prosnier ${ }^{a, b *}$, Vincent Médoc ${ }^{a, b}$, Nicolas Loeuille ${ }^{a}$}

a. Sorbonne Université, Université de Paris, Université Paris-Est Créteil, CNRS, INRAE, IRD, institute of Ecology and Environmental Science of Paris (iEES-Paris), Campus Pierre et Marie Curie, 4 place Jussieu, 75005 Paris, France.

b. Equipe Neuro-Ethologie Sensorielle, ENES/CRNL, CNRS UMR 5292, Université de Lyon/Saint-Etienne, 23 rue Michelon, 42023 Saint-Etienne Cedex 2, France

* Corresponding author. Loïc Prosnier, ENES, Université Jean Monnet - St-Etienne, Campus Métare, Bâtiment K. 21, rue du Dr Paul Michelon 42100 Saint-Etienne, FRANCE. lprosnier@gmail.com

ORCID IDs:

Loïc Prosnier (1prosnier@ gmail.com): 0000-0001-5576-3601

Vincent Médoc (vincent.medoc@univ-st-etienne.fr): 0000-0002-4888-1914

Nicolas Loeuille (nicolas.loeuille@ sorbonne-universite.fr): 0000-0001-9588-6542

\section{Article}

Keyword: Adaptive foraging, virulence, vulnerability, predator diet, profitability, parasitism

Running title: Prey-infection induce diet evolution

Number of words: 5479

List of elements: Article (24 p.) with 1 table and 5 figures. Appendix (4 p.) with 2 figures. 

Parasites, ubiquitous in food webs, are known to affect simultaneously host vulnerability to predation and host energy contents, thereby affecting profitability. In this work, we study the eco-evolutionary consequences of prey infection by a non trophically-transmitted parasite, with a simple lifecycle, on predator diet. We also analyze the consequences for coexistence between prey, predators and parasites. We model a trophic module with one predator and two prey species, one of these prey being infected by a parasite, and distinguish between two effects of infection: a decrease in host fecundity (virulence effect) and an increase in vulnerability to predation (facilitation effect). Predator foraging may evolve toward specialist or generalist strategies, the latter being less efficient on a given resource. We show that the virulence effect leads to specialisation on the non-infected prey while the facilitation effect, by increasing prey profitability, favors specialisation on the infected prey. Combining the two effects at intermediate intensities promotes either generalist predators or the diversification of foraging strategies (coexistence of specialists), depending of trade-off shape. We then investigate how the evolution of predator diet affects the niche overlap between predator and parasite. We show that facilitation effects systematically lead to a high niche overlap, ultimately resulting in the loss of the parasite. Virulence effects conversely favor coexistence by allowing a separation of the predator and parasite niches.

Keyword: Adaptive foraging, virulence, vulnerability, predator diet, profitability, parasitism 


\section{Introduction}

The consequences of parasitism on food webs regarding trophic cascades (Buck and Ripple 2017) or in terms of stabilisation (Hilker and Schmitz 2008; Prosnier et al. 2018) or destabilisation (Hudson et al. 1998; Prosnier et al. 2018) are most often investigated through the lens of ecology. On the other hand, parasite evolution is frequently addressed through the evolution of virulence or transmission, sometimes in a context of trophic interactions (Cressler et al. 2016). However, modulating host phenotype and abundance may also have evolutionary consequences for the species interacting with the hosts, including predators. In this work, we study how infection of a prey species by a specialist parasite with a simple life cycle (i.e. with one host) affects the evolution of the predator foraging strategy.

A possible way to understand variations in predator diet is to consider Optimal Foraging Theory, under which predators interact only with the prey species that enhance their net energy intake rate (Pyke et al. 1977). The relative profitability of prey species, defined as the ratio between energetic gain (prey energetic value) and energetic cost (search and handling times), determines the occurrence of trophic links and therefore the degree of generalism of predators (Emlen 1966; MacArthur and Pianka 1966; Charnov 1976a, 1976b). Although parasite-induced alterations in host phenotype are likely to modify the two components of profitability, the consequences on the structure of natural communities remain poorly understood.

Infection induces modifications in host energy allocation, either because parasites use energy for their own development, or because hosts allocate energy to the immune response at the expense of other functions. This energy reallocation may (negatively) affect host fitness through direct and indirect effects. Direct effects on fitness (virulence effects hereafter) occur when parasites reduce host fecundity, increase host mortality or both. Increased mortality has been described very often (Brassard et al. 1982; Prins and Weyerhaeuser 1987; Lynsdale et al. 2017). 
Infection-induced fecundity reduction is documented for various animals like water fleas (Daphnia sp.) hosting bacterial, viruses or fungi that reduce offspring production by $7-66 \%$ (Decaestecker et al. 2003). Such virulence effects should decrease the density of hosts (prey), which make them less profitable towards predators, search time being increased. Indirect effects on fitness through increased vulnerability to natural enemies and particularly predators (facilitation effects hereafter) results from changes in host phenotype including behavior, morphology or physiology. This can be part of an adaptive transmission strategy in trophicallytransmitted parasites (Cézilly et al. 2010) but also occurs in non-manipulative parasites (Goren and Ben-Ami 2017), as the simple lifecycle parasite without trophic transmission that we consider in this article. For instance, red grouses infected by nematodes are more consumed by mammalian predators due to higher scent emissions, as parasites induce physiological modifications (Hudson et al. 1992). Anisops prefer Daphnia infected by Pasteura ramosa in dark condition but the non-infected Daphnia in light condition (Goren and Ben-Ami 2017), possibly due to modifications in host colouration and mobility. Making the host more attractive (e.g. increased conspicuousness) or more catchable (e.g. decreased mobility) may decrease the search and handling times of predators and thus increase profitability. Note that separating virulence and facilitation effects is a simplification we use to highlight important differences. In reality, both effects may be to some degree correlated: host resources diverted by the parasite for its own development affect simultaneously host fecundity and/or morality (i.e. virulence effect) and host behaviour (i.e. resulting in a facilitation effect). Because virulence and facilitation effects can therefore act in opposite ways on profitability and infection by a single parasite species that induces both effects, the consequences on predator foraging strategies could be unintuitive.

Previous theoretical investigations on the evolution of predator foraging in the absence of parasite suggest that the type of trade-off regarding the relative intensity of predation on each 
prey included in the diet largely affects predator diet evolution (Egas et al. 2004; Rueffler et al. 2006). Concave trade-offs usually select generalist predators (i.e. that consume both prey) whereas convex trade-offs lead to specialist predators (i.e. that consume only one prey) or to diversification (i.e. coexistence of various predator strategies). These works however consider only symmetrical resources (i.e. all prey are identical), so that they cannot account for trait modifications due to parasitism. Infection dynamics can also interact with predation dynamics in complex ways. Here, based on Optimal Foraging, we expect that predators should specialise on the prey whose profitability has been increased by infection. It follows that virulence effects should lead to either generalist strategies or to specialisation on the alternative (i.e. noninfected) prey while specialisation on the infected prey should be observed with facilitation effects.

Beside evolutionary outcomes, we also investigate whether and how the evolution of predator diet affects species coexistence in the trophic module. Coexistence between two prey species depends on a balance between intrinsic competitivity (i.e., which prey species would dominate in the absence of predators and parasites (Gause 1934)) and the intensity of apparent competition (i.e., predator-mediated competitions (Holt 1977)). Prey coexistence is favored when the most competitive species is also the one preferred by the predator (Holt et al. 1994). Because virulence and facilitation effects constrain both direct and apparent competition, the ecological dynamics linked to infection have been shown to influence prey coexistence (Prosnier et al. 2018). Here, we go beyond and tackle the feedback of the evolution of predator diet on these ecological effects with the idea that it may alter apparent competition and thereby prey coexistence in predictable ways. An increase in coexistence is expected in case of virulence effects as evolution should favor the predators that prefer the alternative prey, the negative effects of parasitism being then compensated by a release from predation. Under 
facilitation effects increasing profitability, parasitism should amplify specialisation on the infected species, which may lead to prey exclusion and impair coexistence.

Understanding the conditions of coexistence between parasites and predators requires decomposing the relative effects they have on each other. Regarding the effects of predators on parasites, a classical hypothesis in ecology is that predators may limit parasite prevalence within populations (Healthy Herd Hypothesis, Packer et al. 2003; Lafferty 2004; Duffy et al. 2005) in several ways. First, predators indirectly consume parasites when consuming infected prey (concomitant predation) (Johnson et al. 2010), for instance the signal crayfish (Pacifastacus leniusculus) reduces the abundance (but not the prevalence) of parasites of its isopod and snail prey (Pulkkinen et al. 2013). Second, predators can limit parasite transmission by reducing host density, for instance, Dallas et al. (2018) showed in the water flea Daphnia dentifera that under a density threshold of 80 ind.L-1, its fungal pathogen Metschnikowia bicuspidata cannot be maintained. Such negative effects should be amplified when parasites increase host vulnerability through facilitation effects, as suggested by the models developed by Packer et al. (2003) and Prosnier et al. (2018). Now consider the effects of parasites on the predators of their host. Virulence effects, by reducing host density, decrease predator density through resource limitation (bottom-up effects). Such bottom-up reduction in predator density has for instance been experimentally observed with paramecia infected by Holospora undulata and consumed by Didinum nasutum (Banerji et al. 2015). Conversely, parasites could enhance predator density when they induce facilitation effects, as they increase prey vulnerability (theoretical works of Hethcote et al. [2004] and Prosnier et al. [2018]).

Accounting for the evolution of predator diet should therefore largely influence the outcomes of these ecological effects. If virulence effects promote specialisation on the alternative prey, evolutionary dynamics should lead to niche partitioning between parasites and predators, 
thereby increasing coexistence. On the other hand, facilitation effects should increase niche overlap and thus competition between predators and parasites.

To understand these eco-evolutionary dynamics, we model a trophic module with one predator and two prey in competition, the most competitive prey being infected and structured in susceptible and infected individuals. The parasite induces virulence and facilitation effects, modeled respectively by a reduction of host fecundity or an increase in host vulnerability to predation. In agreement with our predictions, we show that virulence effects favor specialization on the alternative prey while facilitation effects have the opposite effect. Our results also show that evolution favors niche separation among parasites and predators, thereby promoting coexistence.

\section{Model presentation and analysis}

\subsection{Predation-infection model}

The model considers two prey species sharing a predator. We assume both intraspecific and interspecific competition among prey and we model predation through linear functional responses. The infected prey (labeled 1) is also the most competitive and its population is structured in susceptible and infected individuals ( $S_{l}$ and $I_{l}$ respectively). Parasite transmission is horizontal and we assume that infected individuals do not recover. Considering these hypotheses, ecological dynamics follow the set of equations:

$$
\left\{\begin{array}{l}
\frac{d S_{1}}{d t}=S_{1}\left(f_{1}-m_{1}-c_{11} N_{1}-c_{12} N_{2}-a_{1} P\right)+I_{1}\left(\left(f_{1}-n\right)-i S_{1}\right) \\
\frac{d I_{1}}{d t}=I_{1}\left(i S_{1}-c_{11} N_{1}-c_{12} N_{2}-\left(a_{1}+j\right) P-m_{1}\right) \\
\frac{d N_{2}}{d t}=N_{2}\left(r_{2}-c_{21} N_{1}-c_{22} N_{2}-a_{2} P\right) \\
\frac{d P}{d t}=P\left(e a_{1} N_{1}+e j I_{1}+e a_{2} N_{2}-m\right)
\end{array}\right.
$$


bioRxiv preprint doi: https://doi.org/10.1101/2020.04.18.047811; this version posted December 4,2020 . The copyright holder for this preprint (which was not certified by peer review) is the author/funder, who has granted bioRxiv a license to display the preprint in perpetuity. It is made available under aCC-BY-NC-ND 4.0 International license.

, with $N_{x}$ the total prey species $x$ population, $S_{x}$ and $I_{x}$ respectively the susceptible and

139 infected individuals (thus $N_{1}=S_{1}+I_{1}$ ), $f_{1}$ the intrinsic fecundity rate of the infected prey, $m_{1}$

its intrinsic mortality rate, $r_{2}$ is the intrinsic growth rate of the alternative prey (with $r_{2}=f_{2}-$

$\left.m_{2}\right), P$ the predator density, $m$ its intrinsic mortality rate, $i$ the per capita transmission rate of

the parasite, $c_{x x}$ the per capita competition rate of prey $x, c_{x y}$ the per capita competition effect

of prey $y$ on prey $x, a_{x}$ the per capita attack rate of the predator on prey $x, e$ the conversion

efficiency. Virulence effects are implemented through a decreased fecundity in the infected

Prey $N_{1}$ being the most competitive, we assume that $r_{1} c_{22}>r_{2} c_{12}$ et $r_{1} c_{21}>r_{2} c_{11}$ (Hutson parasites.

Table 1. Biological interpretation, dimensions and default values of model's parameters. Used values are those proposed in Prosnier et al. (2018) and based on Hutson and Vickers (1983).

\begin{tabular}{|c|c|c|c|}
\hline Parameters & Descriptions & Defaults values & Dimensions \\
\hline \multicolumn{4}{|c|}{ Model (Eq. (1)) } \\
\hline$N_{1}, N_{2}, P$ & Species densities & - & ind. $m^{-2}$ \\
\hline$S_{1}, I_{1}$ & Density of the susceptible and infected individuals of prey species 1 & - & ind. $m^{-2}$ \\
\hline$f_{1}$ & Intrinsic fecundity rate of prey species 1 & 25 & $d^{-1}$ \\
\hline$m_{1}$ & Intrinsic mortality rate of prey species 1 & 10 & $\mathrm{~d}^{-1}$ \\
\hline$r_{2}$ & Intrinsic growth rate of prey species 2 & 18 & $\mathrm{~d}^{-1}$ \\
\hline$c_{11}$ & Per capita intraspecific competition rate of prey species 1 & 1 & $\mathrm{~m}^{2}$. ind $^{-1} \cdot \mathrm{d}^{-1}$ \\
\hline$c_{22}$ & Per capita intraspecific competition rate of prey species 2 & 8 & $\mathrm{~m}^{2}$. ind $^{-1} \cdot \mathrm{d}^{-1}$ \\
\hline \multirow[t]{2}{*}{$c_{12}$} & Per capita interspecific competition rate of prey species 2 on prey & & $\mathrm{m}^{2}$. ind $^{-1} \cdot \mathrm{d}^{-1}$ \\
\hline & species 1 & 5 & \\
\hline \multirow[t]{2}{*}{$c_{21}$} & Per capita interspecific competition rate of prey species 1 on prey & & $\mathrm{m}^{2} \cdot$ ind $^{-1} \cdot \mathrm{d}^{-1}$ \\
\hline & species 2 & 4 & \\
\hline$a_{1}$ & Per capita attack rate on prey species 1 & - & $\mathrm{m}^{2}$. ind $^{-1} \cdot \mathrm{d}^{-1}$ \\
\hline$a_{2}$ & Per capita attack rate on prey species 2 & - & $\mathrm{m}^{2} \cdot$ ind $^{-1} \cdot \mathrm{d}^{-1}$ \\
\hline$e$ & Conversion efficiency & 1 & Dimensionless \\
\hline$m$ & Predator mortality rate & 2.5 & ind. $\mathrm{m}^{-2} \cdot \mathrm{d}^{-1}$ \\
\hline$i$ & Per capita parasite transmission rate & 35 & $\mathrm{~m}^{2}$. ind $^{-1} \cdot \mathrm{d}^{-1}$ \\
\hline$n$ & Virulence effect (decrease in infected prey fecundity rate) & - & $d^{-1}$ \\
\hline$j$ & Facilitation effect (increase in infected prey vulnerability) & - & $\mathrm{m}^{2}$. ind $^{-1} \cdot \mathrm{d}^{-1}$ \\
\hline \multicolumn{4}{|c|}{ Trade-off (Eq. (2)) } \\
\hline$s$ & Shape of the trade-off & - & Dimensionless \\
\hline$k_{0}$ & Total Allocation in foraging effort & 1.5 & $\mathrm{~m}^{2 \mathrm{~s}} \cdot$ ind $^{-\mathrm{s}} \cdot \mathrm{d}^{-\mathrm{s}}$ \\
\hline
\end{tabular}


The ecological model was previously analyzed by Prosnier et al. (2018). They show that coexistence is possible only given restrictive conditions. Between-prey coexistence is possible

effect). Too large virulence effects allow the parasite to competitively exclude the predator,

while too large facilitation effects allow the predator to overconsume infected prey, thereby leading to the parasite extinction.

\subsection{Trade-off constraints on adaptive foraging}

We assume that the foraging strategy of the predator varies along a trade-off function that

Such trade-offs (Fig. 1) can be modelled using the following function (Egas et al. 2004):

$$
a_{1}^{s}+a_{2}^{s}=k_{0} \leftrightarrow a_{2}=\left(k_{0}-a_{1}^{s}\right)^{1 / s}
$$

, where $s$ affects the trade-off curvature (convex: $s<1$, linear: $s=1$ or concave: $s>1$ ), and $k_{0}$ corresponds to the total allocation of energy or time in predation. 


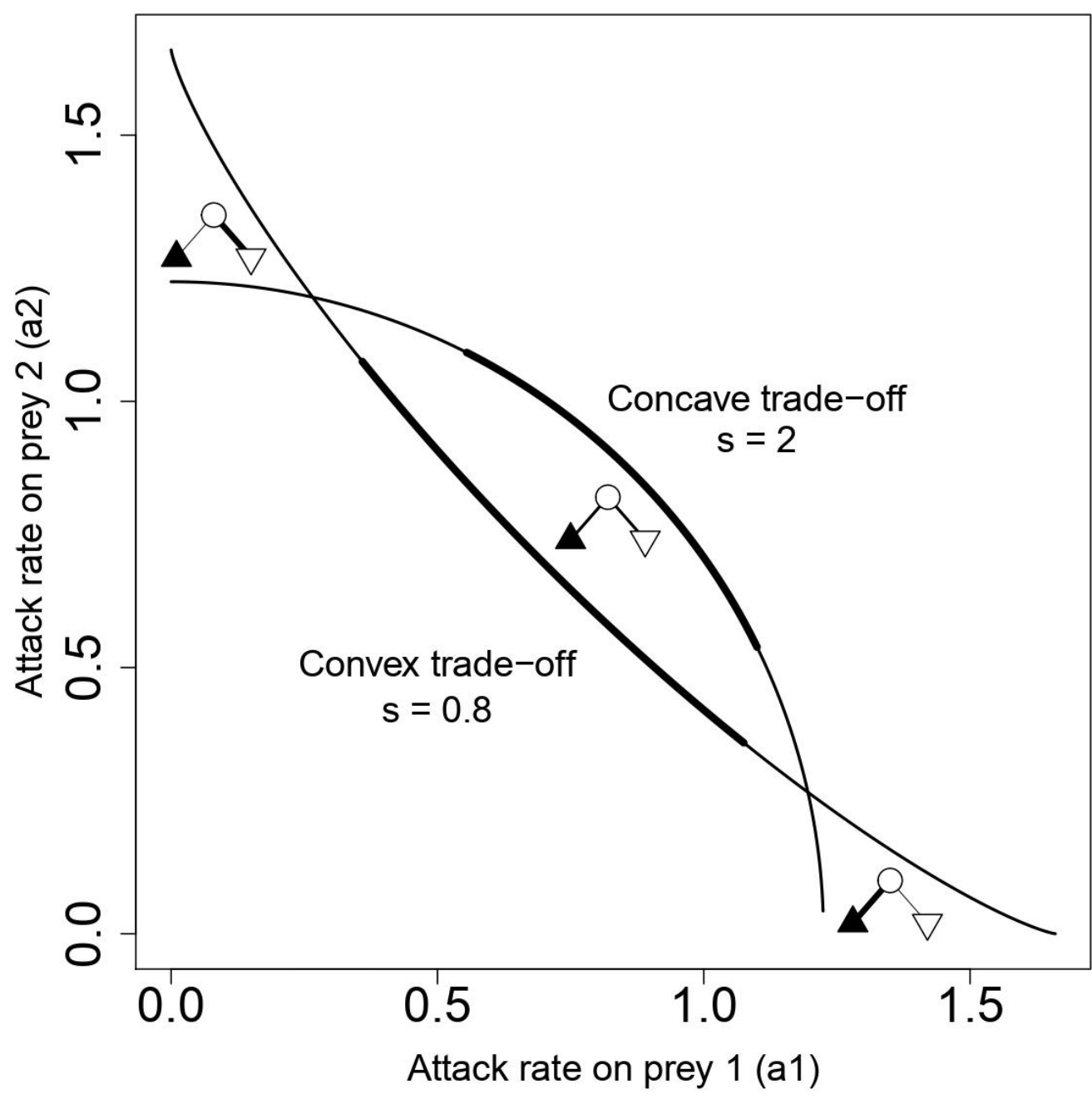

Figure 1. Concave $(s>1)$ and convex $(s<1)$ trade-off of predation investment in each prey. Modules schematize intensity of each trophic link. Symbols represent modules composition: predator (circle), competitive prey (triangle), alternative prey (inversed triangle), infected species are represented in black, healthy species in white. Bold lines show values for generalist predator and thin lines values for specialist predator. See Table 1 for parameter values.

\subsection{Predator diet evolution}

We use adaptive dynamics (Dieckmann and Law 1996; Geritz et al. 1998) to analyse diet evolution (i.e. variations in $a_{1}$ ). Adaptive dynamics assume that evolutionary and ecological such conditions, resident phenotypes $a_{l}$ reach the ecological equilibrium before the next

171 mutation occurs and evolution can be understood based on the invasibility of this equilibrium by nearby mutants (phenotype $a_{1 m}$ ). The relative fitness of mutants can then be defined by their 
173 intrinsic growth rate when rare in these equilibrium conditions (invasion fitness, Metz et al.

$\omega\left(a_{1 m}, a_{1}\right)=\left.\frac{1}{P\left(a_{1 m}\right)} \frac{d P\left(a_{1 m}\right)}{d t}\right|_{\substack{P\left(a_{1 m}\right) \rightarrow 0 \\ P\left(a_{1}\right) \rightarrow P^{*}\left(a_{1}\right)}}$

$\omega\left(a_{1 m}, a_{1}\right)=e a_{1 m}\left(S_{1}{ }^{*}+I_{1}{ }^{*}\right)+e j I_{1}{ }^{*}+e\left(k_{0}-a_{1 m}{ }^{s}\right)^{1 / s} N_{2}{ }^{*}-m$

175 From equation (3), we see that the mutants having high attack rates on prey $N_{1}\left(a_{1 m}\right)$ can

$\frac{d a_{1}}{d t}=\left.\frac{1}{2} \mu \sigma^{2} P^{*}\left(a_{1}\right) \frac{\partial \omega\left(a_{1 m}, a_{1}\right)}{\partial a_{1 m}}\right|_{a_{1 m} \rightarrow a_{1}}$

Where $\mu$ is the per capita mutation rate, $\sigma^{2}$ the phenotypic variance linked to mutation process, and $P^{*}$ the equilibrium density of residents (phenotype $a_{1}$ ). In equation (4), the selection process is described by the slope of the fitness landscape around the resident phenotype, $\left.\frac{\partial \omega\left(a_{1 m}, a_{1}\right)}{\partial a_{1 m}}\right|_{a_{1 m} \rightarrow a_{1}}$. The direction of evolution entirely depends on the sign of this slope, so that $a_{1}$ increases when the slope is positive and decreases when the slope is negative. evolutionary singularities $\bar{a}_{1}$ correspond to:

$$
\left.\frac{\partial \omega\left(a_{1 m}, a_{1}\right)}{\partial a_{1 m}}\right|_{a_{1 m} \rightarrow a_{1} \rightarrow \overline{a_{1}}}=0
$$


invaded by nearby mutants (i.e. non-invasible strategies are ESS). Convergence describes if, starting close to the singularity, selected mutants are even closer to it, so that the strategy is eventually reached. Second derivatives of the fitness $\omega\left(a_{1 m}, a_{l}\right)$ allow us to characterize invasibility and convergence (Diekmann 2004). We define $c_{22}$ as:

$c_{22}=\left.\frac{\partial^{2} \omega}{\partial a_{1 m}^{2}}\right|_{a_{1 m} \rightarrow a_{1} \rightarrow \bar{a}_{1}}$

$c_{22}=-\bar{a}_{1}{ }^{s-2} k_{0}\left(k_{0}-\bar{a}_{1}{ }^{s}\right)^{\frac{1}{s}-2} N_{2}^{*}(s-1)<0$

From this equation we show that non-invasible singularities only occur with a concave tradeoff $(s>1)$.

Each singularity $\bar{a}_{1}$ corresponds to a certain level of foraging generalism, as low values indicate that the predator mostly feeds on the alternative resource, while high values of $\bar{a}_{1}$ indicate that the predator mainly feeds on the host. We consider a predator as a specialist if more than $75 \%$ of its diet is based on one prey (thin lines on Fig. 1).

\subsection{Various outcomes of diet evolution}

In our system, the predator specialises on the most competitive prey $\left(N_{l}\right)$ in the absence of parasite (Not shown). In the presence of parasites, we can observe four outcomes regarding diet evolution, which are depicted on Figures 2, 3 and A1. Figure 2 represents the temporal dynamics of the four populations (uninfected prey, infected prey, non-host prey and predator) and the values of the attack rate on $a_{1}$ - simulations were performed with Scilab (version 5.5.2). Figures 3 and A1 are Pairwise Invasibility Plots (PIP, Diekmann 2004) - that were generated using Mathematica ${ }^{\circledR}$ 11.1.1 (Wolfram research), by plotting the sign of the relative fitness (Equ 

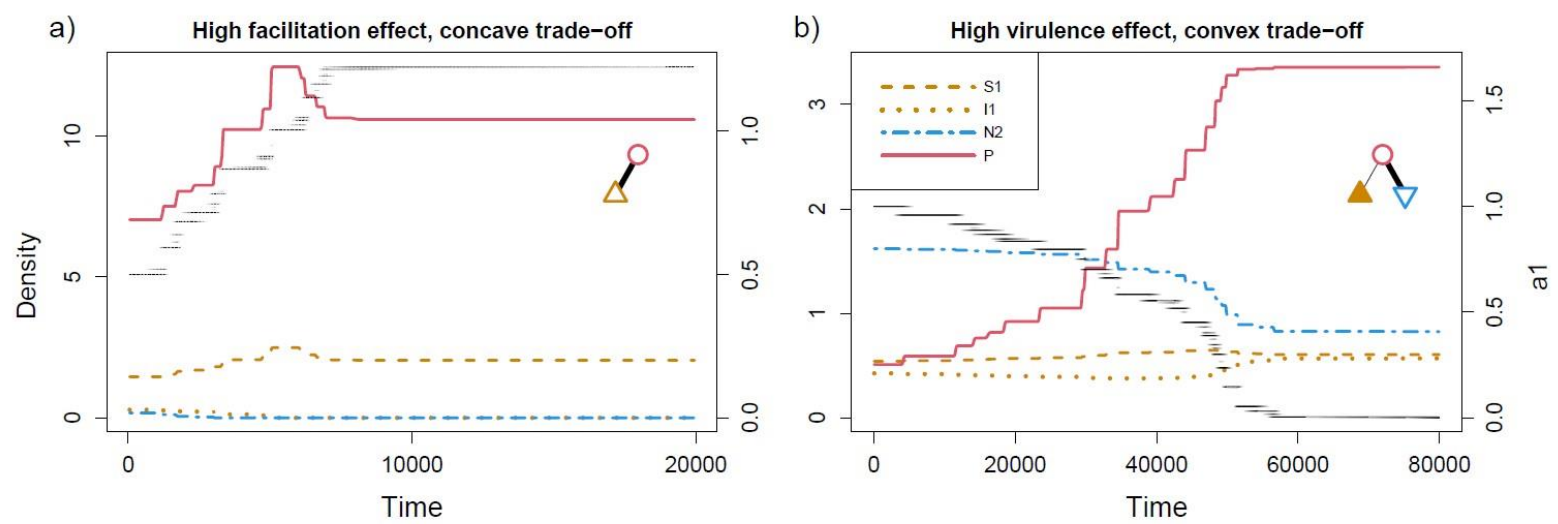

C) Medium virulence and facilitation effects, concave trade-off

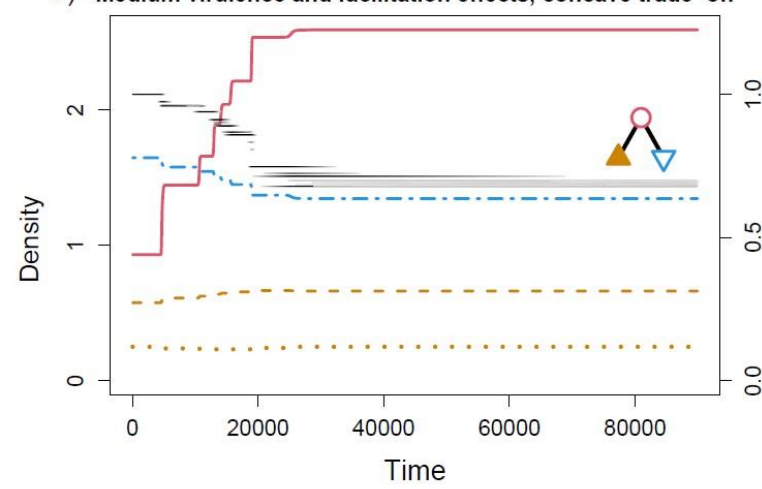

d) Medium virulence and facilitation effects, convex trade-off

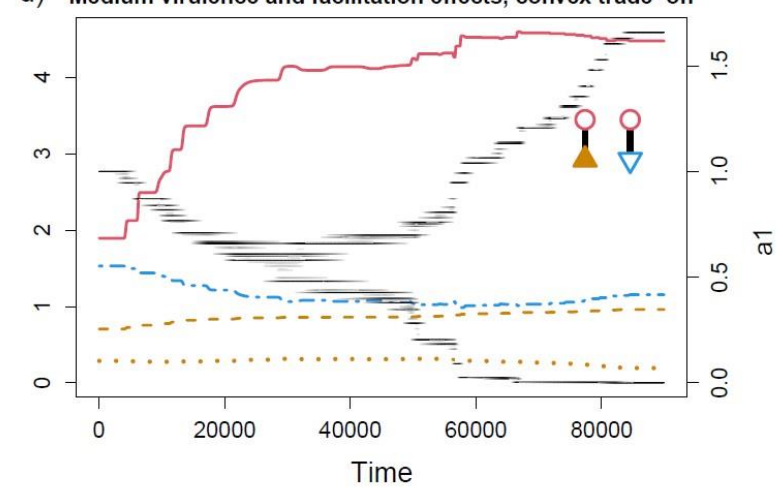

Figure 2. Various eco-evolutionary dynamics (species densities and trait value of the predator) of the module depending of trade-off shape and parasitism effects. a,c) concave trade-off, b,d) convex trade-off. a) high facilitation effect, b) high virulence effect, c,d) medium virulence and facilitation effect. Symbols show the module composition and the intensity of trophic link at the end of the simulation: predator (circle), competitive prey (triangle), alternative prey (inversed triangle). Infected species are show in fill, healthy species in empty. Healthyhost species $S_{l}$ is show in orange dashed line, infected-host species $I_{l}$ in orange dotted line, alternative prey $N_{2}$ in blue dashed-dotted line, predator $P$ in red solid line. Values of $a_{l}$, the evolving trait, is shown in dark (grey scale indicates the density of the predator that have each trait value). Parameter values: see Table 1, except: a) $n=20, j$ $=5, s=2$; b) $n=15, j=2, s=0.8$; c) $n=20, j=2, s=2$; d) $n=15, j=3, s=0.8$.

(3)) when all species equilibriums are positive - , which describe the relative fitness $\omega\left(a_{1 m}, a_{1}\right)$ :

the value of the evolving trait a1 of the resident population (x-axis) relative to that of the mutant

(y-axis). The mutant has a positive relative fitness and thus can invade the population in the dark area, and a negative relative fitness in the white area. Note that because mutations are small and rare, resident and mutant trait values are close to the diagonal $x=y$, and thus the evolution of the trait follows the diagonal, depending of the fitness value above and below the diagonal. Evolutionary directions are shown with white arrows. 
bioRxiv preprint doi: https://doi.org/10.1101/2020.04.18.047811; this version posted December 4, 2020. The copyright holder for this preprint (which was not certified by peer review) is the author/funder, who has granted bioRxiv a license to display the preprint in perpetuity. It is made available under aCC-BY-NC-ND 4.0 International license.

obtain generalist predators with concave trade-off, and diversification and evolutionary

multistability with convex trade-off. In detail, we show that the predator can be generalist (Figs on the host (Fig. 2a) or on the alternative prey (Fig. 2b), which can be achieved in four ways.

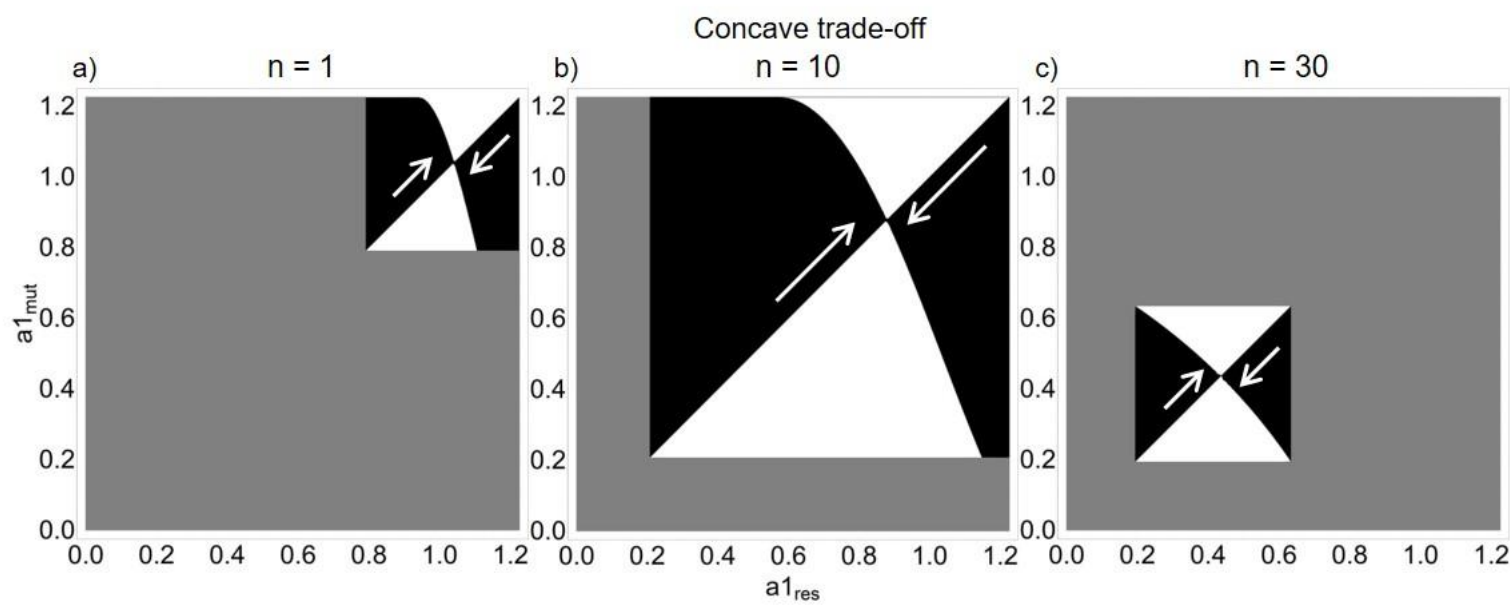

Specialisation on infected prey $\left(\mathrm{N}_{1}\right)$

ce effect

Specialisation on alternative prey $\left(\mathrm{N}_{2}\right)$
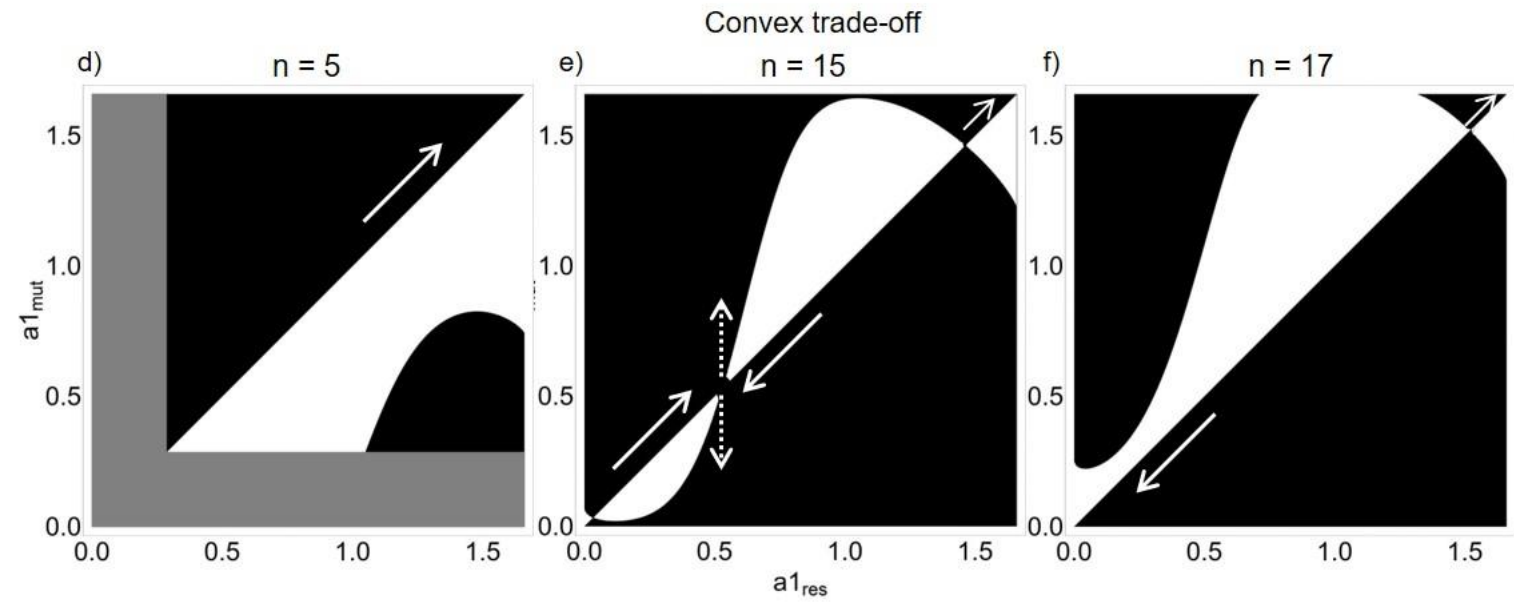

Figure 3. Pairwise Invasibility Plots of evolution of predator diet (i.e. evolution of $a_{l}$ ) for a concave (a-c) and a convex (d-f) trade-offs, when increasing intensity of virulence effect. On PIP, black area corresponds to a positive relative fitness of mutants, white area corresponds to a negative relative fitness of mutants, grey area shows nocoexistence of the system. The white solid arrows show the direction of evolution, the white dotted arrows show evolutionary branching. a-c) CSS, d) no singularity, e) one EBP and two repellors, f) one repellor. Parameter values: see Table 1, except a) $n=1, j=1, s=2$; b) $n=10, j=1, s=2$; c) $n=30, j=1, s=2$; d) $n=5, j=3, s=$ 0.8 ; e) $n=15, j=3, s=0.8$; f) $n=17, j=3, s=0.8$. Note that Fig A1 shows the results with the facilitation effect. 
224 First, specialists could correspond to CSS of large or small values (Fig. 3a,c, A1a).

Alternatively, the singularity may be a Repellor (i.e. non-convergent and invasible), with evolution leading to specialization on one species or another depending on the initial diet. In our model, repellors only occur with convex trade-offs as they are invasible (eq. 7) (Fig. 3f). A last possibility to obtain a specialist is that no singularity exists, when the fitness gradient (eq. 3 ) is always positive or negative. Evolution then leads to a continuous increase or decrease of $a_{1}$, so that complete specialization is selected (Fig. 3d, A1d,f). Differentiating the fitness function (3) to get the fitness gradient, it can easily be shown that the loss of one prey systematically selects specialization on the other prey (Fig. 2a). Finally, we can obtain a diversification of the predator strategies (polymorphism) with the coexistence of two specialists, one on each prey (Figs $2 \mathrm{~d}, 3 \mathrm{e}, \mathrm{A} 1 \mathrm{e}$ ). Such evolutionary dynamics correspond to Evolutionary Branching Points (EBP): a singularity that is convergent and invasible. Such branchings occur only for convex trade-offs where the singularity is invasible (eq. 7). Note that these outcomes are not mutually exclusive, as several singularities may coexist for a given set of parameters, yielding complex evolutionary dynamics. For instance, on Fig. 3e, two repellors coexist with a branching point, so that, depending of its initial diet, a predator can evolve to specialisation on the host (if the initial $a_{1}$ is high, i.e. higher than the value of the highest repellor), on the alternative prey (if the initial $a_{1}$ is low, i.e. lower than the value of the lowest repellor), or to diversification (i.e. a coalition of two specialists as illustrated on Fig. 2d) (Fig. $3 e$ and $\mathrm{A} 1 \mathrm{e})$.

\subsection{Parasites affect diet evolution}

Now that we have described the possible outcomes, we investigate how the intensity of the parasite-induced virulence and facilitation effects constrains their occurrence. Virulence effects are depicted on Fig. 3, facilitation effects on Fig. A1 and both effects investigated concurrently on Fig. 4 - Data were generated with Mathematica ${ }^{\circledR}$, using analytical form of the fitness 
gradient and the equilibriums of the four species: for each parameters couple we obtain the numerical value of the fitness and then search the zero-crossing along an $a_{1}$ gradient; we reported either the corresponding value (for a null fitness) of $a_{l}$ for the concave trade-off, either the number of zero-crossing for the convex trade-off; for each zero-crossing we control for the existence of all species.

When only virulence effects occur (Fig. 3), as expected, a higher virulence changes the outcome of evolution from specialisation on the infected prey to specialisation on the alternative prey. Conversely, but also in agreement with our predictions, higher facilitation effects eventually lead to specialisation on the infected prey (Appendix A). In both cases, intermediate effects either lead to generalist strategies (concave trade-offs, Fig. 3b, A1b), or to diversification (convex trade-offs, Fig. 3e, A1e). To go further and assuming concave trade-offs, increasing the virulence effects move the CSS toward lower values of $a_{1}$, so that the predator increasingly specializes on the alternative prey (Fig. 3a-c), while increasing facilitation effects move the CSS toward higher values of $a_{1}$ so that the predator increasingly relies on the host species (Fig. A1a-c). Similarly, for convex trade-offs, higher virulence effects move the system from a complete specialization on the host (Fig. 3d) to a situation in which most dynamics would lead to specialization on the alternative prey (fig. 3f). Analogous variations can be described when decreasing the facilitation effects (fig. A1d-f).

Figure 4 summarizes these antagonistic consequences of virulence and facilitation effects, and how they depend on the shape of the trade-off function. As previously described, specialist strategies are selected when either virulence or facilitation effects are strong (Fig. 4, light grey and black areas), regardless of the shape of the trade-off. However, most parasites are likely to induce virulence and facilitation effects simultaneously, so that the evolutionary outcome may often be in between these extreme cases (i.e. in the middle of the panels of Fig. 4). Such evolutionary outcomes depend on the shape of the trade-off. Concave trade-offs allow 


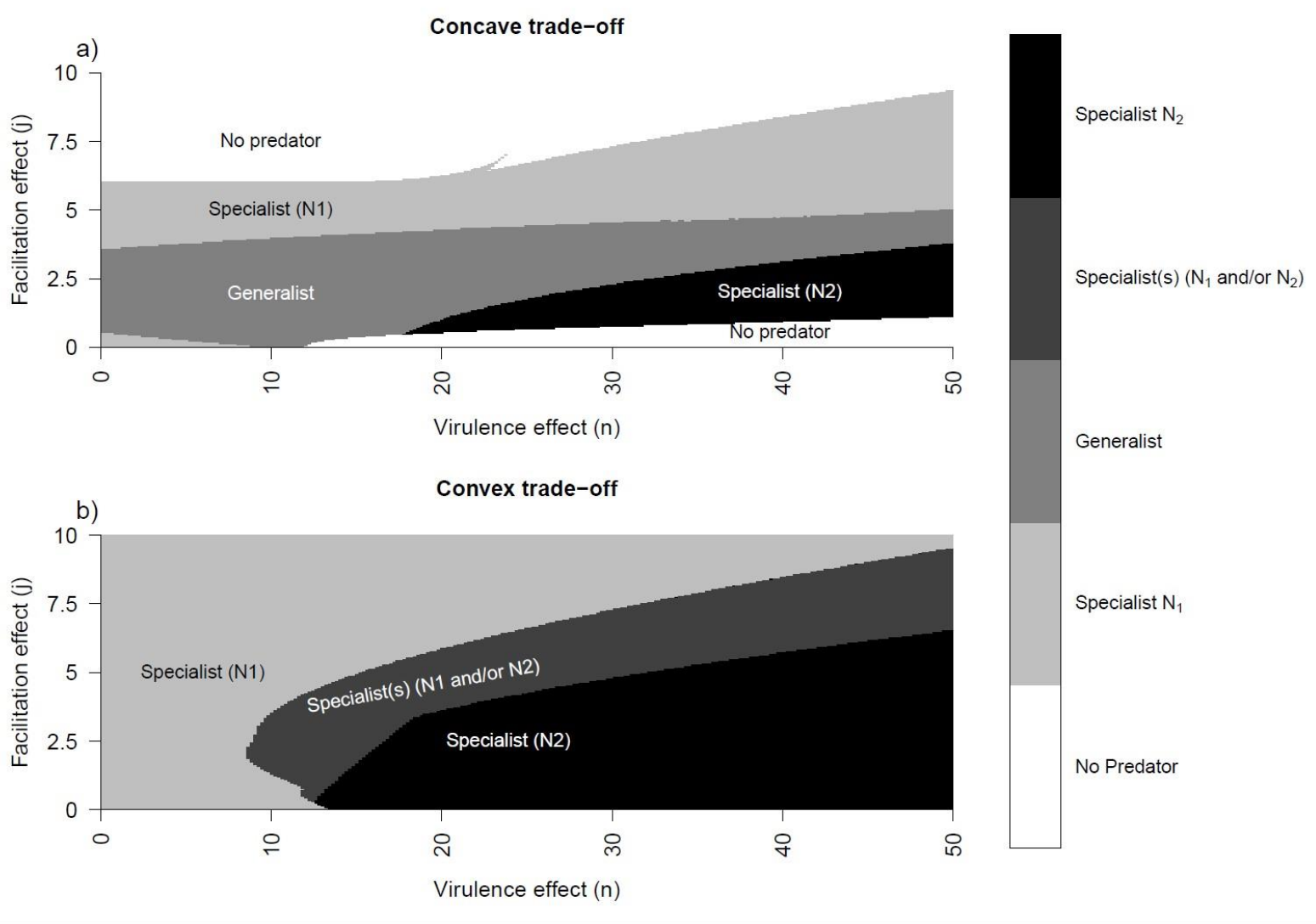

Figure 4. Predator diet after evolution, function of virulence effect (x-axis) and facilitation effect (y-axis) of the parasite, with concave (a) and convex (b) trade-offs. Parameter values: see Table 1, except a) $\mathrm{s}=2, \mathrm{~b}$ ) $\mathrm{s}=0.8$.

generalist strategies (medium grey, Fig. 4a) while convex trade-offs favor the diversification of predator diets, potentially leading to the coexistence of specialist strategies (dark grey, Fig. 4b).

Finally, note on Figure 4 that extreme effects may lead to the loss of coexistence within the module (white areas), highlighting the fact that evolutionary responses of predators to prey infection may have far reaching consequences for community structure. We now analyze such consequences for species coexistence in further details.

\subsection{Consequences on coexistence}

While we know that parasitism may directly constrain coexistence of prey by changing the relative weight of direct and apparent competition (Prosnier et al. 2018), here we highlight that evolutionary dynamics in response to parasitism may favor coexistence in trophic modules. To clarify the role of evolution, on figure 5, we show the coexistence conditions with (grey area) and without (area delimited by the solid line) diet evolution. For the "without evolution" 
scenarios, we consider as reference cases in which the predator is specialised on the infected

prey (Fig. 5a), on the alternative prey (Fig. 5c), or is a generalist (Fig. 5b).

Our results suggest that evolution systematically enhances the size of coexistence area. The coexistence area is increased by a factor 2.22 compared to a predator specialist on the host prey

a)

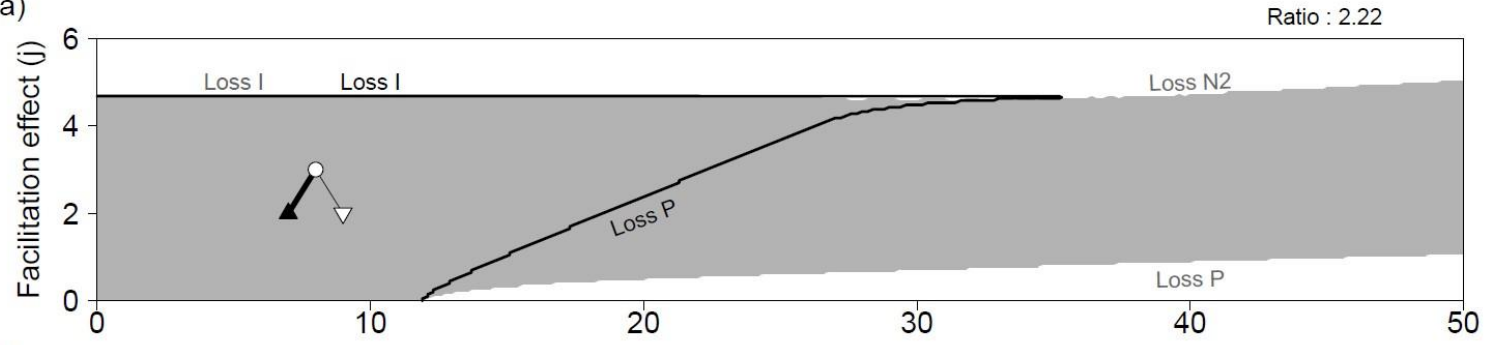

b)

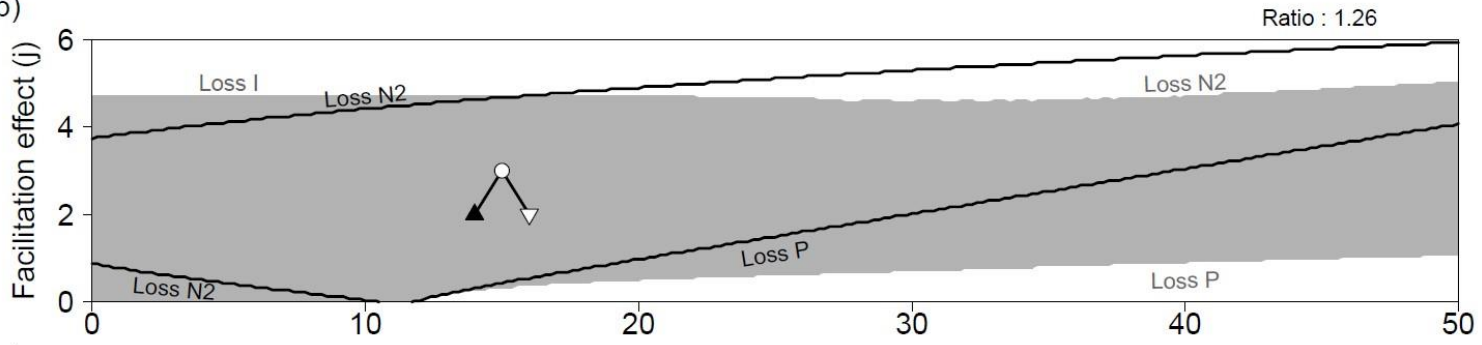

c)

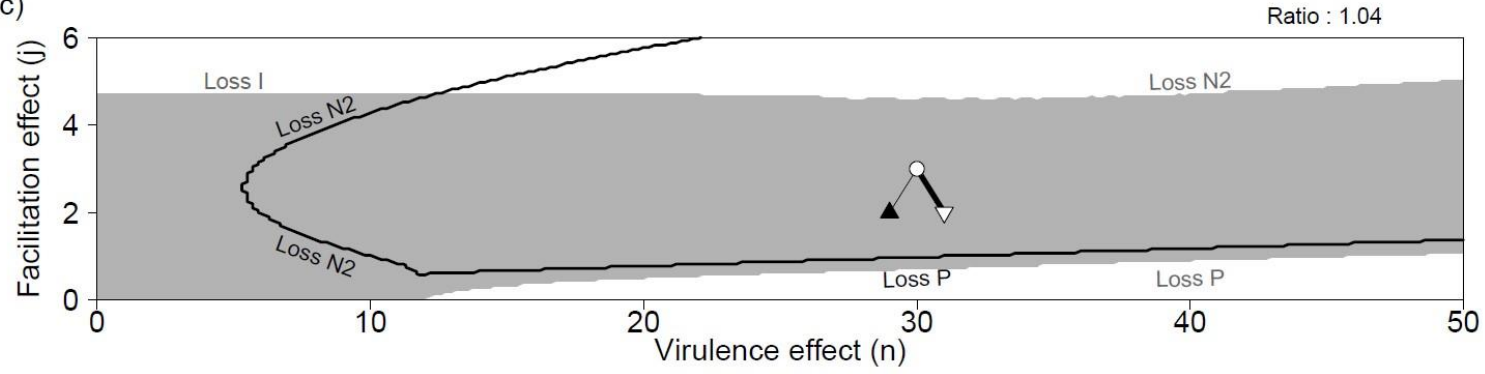

Figure 5. Coexistence of the three species, with convex trade-off, with evolution (grey area) and without evolution (inside the black delimitation). When the predator is a) specialist on the infected prey $N_{1}, \mathrm{~b}$ ) generalist, c) specialist on the alternative prey $N_{2}$. The species loss at the limits are written, in dark without evolution, in grey with evolution. Ratio is the ratio of coexistence surface with/without evolution. Parameters value: see Table 1 except $s=2$. Note that Fig B1 shows the results with a concave trade-off. 
predator is excluded because the parasite decreases the amount of prey available, especially in case of specialisation on the host prey (Fig. 5a). Here evolution promotes coexistence in that it favors the diets more oriented toward the alternative prey. Note that while the global effect of evolution is to enhance coexistence, it may decrease it for particular effects. For instance, when both virulence and facilitation effects are high, from an ecological point of view a balance exists between apparent and direct competition among prey and coexistence is possible without evolution (e.g. Fig. 5b,c, see also Prosnier et al. 2018). Evolution however increases the weight of apparent competition and results in the exclusion of the alternative prey. While Fig. 5 assumes a concave trade-off, we note that the effects of evolution on coexistence are similar for convex trade-offs (see Appendix B).

\section{Discussion}

Parasites likely affect food webs in many ways. Because of their virulence and the resulting decrease in fecundity, they can constrain the availability of prey populations, thereby leading to resource limitation for predators. Alternatively, parasite-induced phenotypic alterations can strengthen trophic interactions, making infected prey more susceptible to predation. While these ecological consequences of parasitism for food webs have received some attention (McNaughton 1992; Banerji et al. 2015; Buck and Ripple 2017; Buck 2019), parasitism also affects coevolution between prey and predator species. Here, our model shows that the evolution of predator diet depends on the type of parasitism effect (virulence or facilitation) and its intensity. We show that virulence effects usually lead to the selection of increased predation on the alternative (i.e. non host) prey, while facilitation effects favor predation on the host species. Such results are consistent with our predictions based on host profitability: virulence effects reduce profitability whereas facilitation effects increase it. Evolution also favors coexistence among prey and between predators and parasites. 
As expected, virulence and facilitation effects have antagonistic consequences on the evolution of predator diet. Virulence effects (i.e. decreased host fecundity) lead to specialization

322

on the alternative prey. Because virulence decreases the host prey population, the first part of the fitness function (Eq. 2) is decreased and foraging strategies that focus more on the alternative prey are favored. Conversely, facilitation effects (i.e. increase in host vulnerability) lead to specialization on the infected prey. Again, turning to the definition of fitness (Eq. 2), the first term is increased when facilitation effects are present, so that strategies focusing more on the host species are selected. Consequently, the evolution of predator diet can here be understood in the context of optimal foraging (Emlen 1966; MacArthur and Pianka 1966; Charnov 1976a, 1976b). Predators can be generalists when virulence and facilitation effects have intermediate intensities and/or when they act simultaneously. Thus, we expect diet to shift progressively from specialism on one prey to the other prey when the two effects vary. Generalism requires balanced virulence and facilitation effects and concave trade-offs. It is replaced by a coalition of two specialists given convex trade-offs. Such a link between tradeoff shape and diversification is consistent with previous theoretical works on the evolution of specialization (Egas et al. 2004; Rueffler et al. 2006; van Velzen and Etienne 2013).

The evolution of predator diet influences coexistence between the two prey species, because it depends on the balance between direct and apparent competition. It also influences the predator-parasite coexistence, by modulating the resource overlap between the two species. Concerning the coexistence of prey species, virulence effects reduce host competitivity and may ultimately lead to its exclusion (Prosnier et al. 2018). With evolution, however, a decrease in host density selects a lower consumption by the predator, which balances the decrease in host competitive ability thereby favoring its persistence. Similarly, if the alternative prey were to become rare, evolution would select diets that are more focused on the host prey, so that coexistence would again be favored. In a nutshell, adaptive foraging creates a negative 
frequency-dependence that promotes the persistence of the rare prey, a mechanism already pointed out by several works (Kondoh 2003; Křivan and Eisner 2003; Loeuille 2010) but not to our knowledge in the context of parasitism. Conversely, given facilitation effects, evolution selects higher predation on the host, so that the negative effects of parasitism on prey coexistence are magnified (i.e. both parasitism and evolution increase predation on the host prey).

We observe an increase in predator-parasite coexistence due to niche partitioning between parasite and predator when parasites induce virulence effects. This is consistent with approaches that unify parasite and predator under the same natural enemy concept (Raffel et al. 2008). Here, the parasite and the predator can be viewed as intraguild predators since they compete for the same trophic resource (i.e. the host population) while the predator also kills the parasite through concomitant predation (i.e. when it consumes infected individuals) (Sieber and Hilker 2011). Niche partitioning between intraguild predators due to evolutionary dynamics has already been shown (Ingram et al. 2012). While the widespread co-occurrence of parasites and predators in food webs (Lafferty et al. 2006) can be puzzling given the competition that exists between the two groups, our results on the role of diet evolution may offer a possible explanation. Stewart et al. (2018) described what could be interpreted as niche partitioning between the parasites and predators of Ceriodaphnia in the lake Gatun (Panama). However, direct comparison with our theoretical work is difficult as the evolutionary processes remain unknown. An empirical validation of our results would involve experimental works based on short-lived organism to allow rapid evolution. For instance, that could be the system used by Banerji et al. (2015): Paramecium caudatum infected by Holospora undulata and consumed by a rotifer, Didinium nasutum, which allows ecological dynamics in less than two months. We could add a competitor to $P$. caudatum through another species of Paramecium or another ciliate like Stylonychia pustulata as done by Gause (1934). 
In this work, we show how parasites may affect the evolution of predator diet and how in return adaptive foraging alters species coexistence. How such relationship between evolution of foraging and parasitism affects more complex food webs would be a natural extension of the present work. For instance, while coexistence of predators and parasites may not be easily explained from an ecological point of view (due to competition), our results suggest that evolution can provide the niche partitioning needed to allow the maintenance of their diversity and could explain why "a healthy system to be one that is rich in parasite species" (Hudson et al. 2006). However, at this point, it seems premature to extrapolate our results to complex food webs, as empirical information is lacking for several important aspects. We chose to discuss two points: the functional response and the predator infection.

Because we aimed at studying a variety of evolutionary scenarios (flexible trade-offs, different effects of parasitism), we voluntarily simplified the ecological dynamics. We for instance use a type-I functional response. While type-II functional responses are sometimes preferred, it is unclear, in the case of facilitation effects of the parasite, which parameters of the functional response would be modified (i.e. handling times or attack rates). Also, we expect that our main results concerning the effect of adaptive foraging on species coexistence and predator-parasite niche separation to be robust. For instance, given a type II function, it is easy to show that when the infected prey is low due to parasitism effects, evolution will still favor consuming the alternative prey, thereby promoting prey coexistence and niche separation, as in the type I case. These results are consistent with literature that suggest that adaptive foraging creates a frequency-dependent process that is utterly stabilizing, regardless of the functional response used (Loeuille 2010; Valdovinos et al. 2010). infected by the parasite when it eats an infected prey - i.e. a trophic transmission (see for 
instance the model of Fleischer et al. (2020), where predator evolve in response to trophicallytransmitted parasites). Considering our results, if parasites have also a virulent effect on the predator, we should expect that it magnifies the niche separation, because the parasite should be both a competitor and a parasite for the predator. However, in some well-known cases niche separation is not possible: when the predator is the final host (i.e. parasites with complex cycle).

Because parasites then need to infect the predator to complete their cycle, we deduce from our results some constraints on the virulence and the facilitation effects due to the evolution of predator diet. The virulence should be lower both for the prey and the predator (see the model of Kuris, 2003, for a low virulence for the predator, but not for the prey, and the review of Fayard et al. (2020) for a low virulence on the intermediate host). However, if avirulence is not possible (due to evolutionary or biological constraints), the parasite should maximize the facilitation effect (i.e. the intermediate host should be prey by the final host before its death). coexistence between the predator and the parasite, contrary to our system where such situations would lead to parasite extinction. Moreover, it should be beneficial to the predator (Øverli and Johansen 2019). Consequently, we show from our work and the resulting discussion, that studying facilitation effects is also interesting for non trophically-transmitted parasites, and virulence effects for trophically-transmitted parasites. However, and despite their nonindependence, both lacking in past works.

Finally, despite some simplifications of our model, the possible generalization of the results and the various scenarios studied allow us to consider possible effects of parasitism in food webs that seem currently understudied. Our study should be a base both for empirical works, as detailed by Fleischer et al. (2020) on the stickleback model that could prey on benthic and/or 418 pelagic prey variously infected, and for theoretical works on the complex implications of parasitism on food webs. 


\section{References}

Banerji, A., A. B. Duncan, J. S. Griffin, S. Humphries, O. L. Petchey, and O. Kaltz. 2015. Densityand trait-mediated effects of a parasite and a predator in a tri-trophic food web. Journal of Animal Ecology 84:723-733.

Brassard, P., M. E. Rau, and M. A. Curtis. 1982. Infection dynamics of Diplostomum spathaceum cercariae and parasite-induced mortality of fish hosts. Parasitology 85:489-493.

Buck, J. C. 2019. Indirect Effects Explain the Role of Parasites in Ecosystems. Trends in Parasitology $35: 835-847$.

Buck, J. C., and W. J. Ripple. 2017. Infectious Agents Trigger Trophic Cascades. Trends in Ecology \& Evolution 32:681-694.

Cézilly, F., F. Thomas, V. Médoc, and M.-J. Perrot-Minnot. 2010. Host-manipulation by parasites with complex life cycles: adaptive or not? Trends in Parasitology 26:311-317.

Charnov, E. L. 1976a. Optimal Foraging: Attack Strategy of a Mantid. The American Naturalist 110:141-151.

. 1976b. Optimal foraging, the marginal value theorem. Theoretical population biology 9:129 136.

Cressler, C. E., D. V McLeod, C. Rozins, J. Van Den Hoogen, and T. Day. 2016. The adaptive evolution of virulence: a review of theoretical predictions and empirical tests. Parasitology 143:915930.

Dallas, T. A., M. Krkošek, and J. M. Drake. 2018. Experimental evidence of a pathogen invasion threshold. Royal Society Open Science 5:171975.

Decaestecker, E., A. Vergote, D. Ebert, and L. De Meester. 2003. Evidence for strong host cloneparasite species interactions in the daphnia microparasite system. Evolution 57:784-792.

Dieckmann, U., and R. Law. 1996. The dynamical theory of coevolution: a derivation from stochastic ecological processes. Journal of mathematical biology 34:579-612.

Diekmann, O. 2004. Beginner's Guide to Adaptive Dynamics. Banach Center Publications 63:47-86.

Duffy, M. A., S. R. Hall, A. J. Tessier, and M. Huebner. 2005. Selective predators and their parasitized prey: Are epidemics in zooplankton under top-down control? Limnology and Oceanography 50:412420.

Egas, M., U. Dieckmann, and M. W. Sabelis. 2004. Evolution restricts the coexistence of specialists and generalists: the role of trade-off structure. The American naturalist 163:518-531.

Emlen, J. M. 1966. The Role of Time and Energy in Food Preference. The American Naturalist 100:611-617.

Eshel, I. 1983. Evolutionary and Continnoas Stability. Journal of Theoretical Biology 103:99-111.

Fayard, M., F. Dechaume-Moncharmont, R. Wattier, and M. Perrot-Minnot. 2020. Magnitude and direction of parasite-induced phenotypic alterations: a meta-analysis in acanthocephalans. Biological Reviews 95.

Fleischer, S. R., D. I. Bolnick, and S. J. Schreiber. 2020. Sick of Eating : eco-evo-immuno dynamics of predators and their trophically acquired parasites. bioRxiv 1-31. 
Gause, G. F. 1934. The struggle for existence. The Williams \& Wilkins Company.

Geritz, S. A. H., É. Kisdi, G. Meszéna, and J. A. J. Metz. 1998. Evolutionarily singular strategies and the adaptive growth and branching of the evolutionary tree. Evolutionary Ecology 12:35-57.

Geritz, S. A. H., J. A. J. Metz, É. Kisdi, and G. Meszéna. 1997. Dynamics of Adaptation and Evolutionary Branching. Physical Review Letters 78:2024-2027.

Goren, L., and F. Ben-Ami. 2017. To eat or not to eat infected food: a bug's dilemma. Hydrobiologia 798:25-32.

Hethcote, H. W., W. Wang, L. Han, and Z. Ma. 2004. A predator - Prey model with infected prey. Theoretical Population Biology 66:259-268.

Hilker, F. M., and K. Schmitz. 2008. Disease-induced stabilization of predator-prey oscillations. Journal of Theoretical Biology 255:299-306.

Holt, R. D. 1977. Predation, apparent competition, and the structure of prey communities. Theoretical population biology 12:197-229.

Holt, R. D., J. Grover, and D. Tilman. 1994. Simple Rules for Interspecific Dominance in Systems with Exploitative and Apparent Competition. The American Naturalist 144:741-771.

Hudson, P. J., A. P. Dobson, and K. D. Lafferty. 2006. Is a healthy ecosystem one that is rich in parasites? Trends in Ecology \& Evolution 21:381-385.

Hudson, P. J., A. P. Dobson, and D. Newborn. 1992. Do Parasites make Prey Vulnerable to Predation? Red Grouse and Parasites. The Journal of Animal Ecology 61:681.

Hudson, P. J., A. P. Dobson, and D. Newborn. 1998. Prevention of Population Cycles by Parasite Removal. Science 282:2256-2258.

Hutson, V., and G. T. Vickers. 1983. A criterion for permanent coexistence of species, with an application to a two-prey one-predator system. Mathematical Biosciences 63:253-269.

Ingram, T., R. Svanbäck, N. J. B. Kraft, P. Kratina, L. Southcott, and D. Schluter. 2012. Intraguild predation drives evolutionary niche shift in threespine stickleback. Evolution 66:1819-1832.

Johnson, P. T. J., A. P. Dobson, K. D. Lafferty, D. J. Marcogliese, J. Memmott, S. A. Orlofske, R. Poulin, et al. 2010. When parasites become prey: ecological and epidemiological significance of eating parasites. Trends in Ecology \& Evolution 25:362-371.

Kondoh, M. 2003. Foraging Adaptation and the Relationship Between Food-Web Complexity and Stability. Science 299:1388-1391.

Křivan, V., and J. Eisner. 2003. Optimal foraging and predator-prey dynamics III. Theoretical Population Biology 63:269-279.

Kuris, A. M. 2003. Evolutionary Ecology of Trophically Transmitted Parasites. Journal of Parasitology 89:S96-S100.

Lafferty, K. D. 2004. Fishing for lobsters indirectly increases epidemics in sea urchins. Ecological Applications 14:1566-1573.

Lafferty, K. D., A. P. Dobson, and A. M. Kuris. 2006. Parasites dominate food web links. Proceedings of the National Academy of Sciences 103:11211-11216.

Loeuille, N. 2010. Consequences of adaptive foraging in diverse communities. Functional Ecology 24:18-27. 
Lynsdale, C. L., H. S. Mumby, A. D. Hayward, K. U. Mar, and V. Lummaa. 2017. Parasite-associated mortality in a long-lived mammal: Variation with host age, sex, and reproduction. Ecology and Evolution 7:10904-10915.

MacArthur, R. H., and E. R. Pianka. 1966. On Optimal Use of a Patchy Environment. The American Naturalist 100:603-609.

McNaughton, S. J. 1992. The propagation of disturbance in savannas through food webs. Journal of Vegetation Science 3:301-314.

Metz, J. A. J., R. M. Nisbet, and S. A. H. Geritz. 1992. How should we define "fitness" for general ecological scenarios? Trends in ecology \& evolution 7:198-202.

Øverli, Ø., and I. B. Johansen. 2019. Kindness to the Final Host and Vice Versa: A Trend for Parasites Providing Easy Prey? Frontiers in Ecology and Evolution 7.

Packer, C., R. D. Holt, P. J. Hudson, K. D. Lafferty, and A. P. Dobson. 2003. Keeping the herds healthy and alert: implications of predator control for infectious disease. Ecology Letters 6:797-802.

Prins, H. H. T., and F. J. Weyerhaeuser. 1987. Epidemics in Populations of Wild Ruminants: Anthrax and Impala, Rinderpest and Buffalo in Lake Manyara National Park, Tanzania. Oikos 49:28-38.

Prosnier, L., V. Médoc, and N. Loeuille. 2018. Parasitism effects on coexistence and stability within simple trophic modules. Journal of Theoretical Biology 458:68-77.

Pulkkinen, K., T. J. Ruokonen, M. Mykrä, G. Tambe, J. Karjalainen, and H. Hämäläinen. 2013. Indirect effects of invasive crayfish on native fish parasites. Ecosphere 4:art50.

Pyke, G. H., H. R. Pulliam, and E. L. Charnov. 1977. Optimal Foraging: A Selective Review of Theory and Tests. The Quarterly Review of Biology.

Raffel, T. R., L. B. Martin, and J. R. Rohr. 2008. Parasites as predators: unifying natural enemy ecology. Trends in Ecology \& Evolution 23:610-618.

Rueffler, C., T. J. M. Van Dooren, and J. A. J. Metz. 2006. The Evolution of Resource Specialization through Frequency-Dependent and Frequency-Independent Mechanisms. The American Naturalist 167:81-93.

Sieber, M., and F. M. Hilker. 2011. Prey, predators, parasites: intraguild predation or simpler community modules in disguise? Journal of Animal Ecology 80:414-421.

Stewart, T. E., M. E. Torchin, and C. E. Cáceres. 2018. Invisible Parasites and Their Implications for Coexisting Water Fleas. Journal of Parasitology 104:101-105.

Valdovinos, F. S., R. Ramos-Jiliberto, L. Garay-Narváez, P. Urbani, and J. A. Dunne. 2010. Consequences of adaptive behaviour for the structure and dynamics of food webs. Ecology Letters 13:1546-1559.

van Velzen, E., and R. S. Etienne. 2013. The evolution and coexistence of generalist and specialist herbivores under between-plant competition. Theoretical Ecology 6:87-98. 
bioRxiv preprint doi: https://doi.org/10.1101/2020.04 18.047811; this version posted December 4, 2020. The copyright holder for this preprint (which was not certified by peer review) is the author/funder, who has granted bioRxiv a license to display the preprint in perpetuity. It is made available under aCC-BY-NC-ND 4.0 International license.

\section{Appendix}

\section{A) Diet evolution due to parasitism: facilitation effect}

When only facilitation effects occur (Fig. A1), a higher facilitation effect always leads


Figure A1. Pairwise Invasibility Plots of evolution of predator diet (i.e. evolution of $a_{1}$ ) for a concave (a-c) and a convex (d-f) trade-offs, when increasing intensity of facilitation effect. On PIP, black area corresponds to a positive relative fitness of mutants, white area corresponds to a negative relative fitness of mutants, grey area shows nocoexistence of the system. The white solid arrows show the direction of evolution, the white dotted arrows show evolutionary branching. a-c) CSS, d) no singularity, e) one EBP and two repellors, f) no singularity. Parameter values: see Table 1, except a) $n=20, j=45, s=2$; b) $n=20, j=30, s=2$; c) $n=20, j=15, s=2$; d) $n=15, j=$ $5, s=0.8$; e) $n=15, j=3.1, s=0.8$; f) $n=15, j=1, s=0.8$. Note that Fig 3 shows the results with the virulence effect. 
541 (concave trade-offs, A1b), or to diversification (convex trade-offs, A1e). To go further and

542 assuming concave trade-offs, increasing the facilitation effects move the CSS toward higher

543 values of $a_{1}$, so that the predator increasingly specializes on the infected prey (Fig. 1Aa-c).

544 Similarly, for convex trade-offs, higher virulence effects move the system from a complete

545 specialization on the alternative prey (Fig. A1f), to a situation in which most dynamics would

546 converge to diversification (i.e. the coexistence of two specialists, Fig. A1e), eventually leading

547 to situations in which almost all initial diets will lead to specialization on the host (fig. A1d).

548 However, note that, in some case, as on Fig. A1d if you start with $0.1<a_{1}<1$ you may loss

549 coexistence of the system through the loss of the parasite. 


\section{B) Consequences on coexistence: concave trade-off}

Similarly, as for convex trade-off, we compare the coexistence conditions with (grey area) and without (area delimited by the solid line) diet evolution. For the "without evolution" scenarios, we consider the cases where the predator is specialised on the infected prey (Fig. B1a), the alternative prey (Fig. B1c), or is a generalist (Fig. B1b).

Our results show that coexistence with evolution is higher than coexistence without evolution. The coexistence area is increased by a factor 3.58 compared to a predator specialist predation and competition could result in the exclusion of the alternative prey (Fig. B1c),

a)

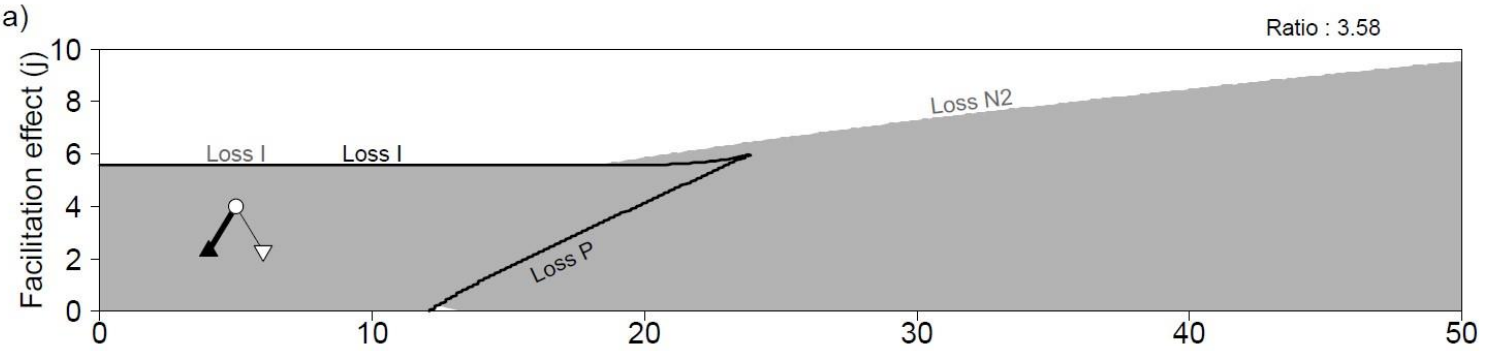

b)

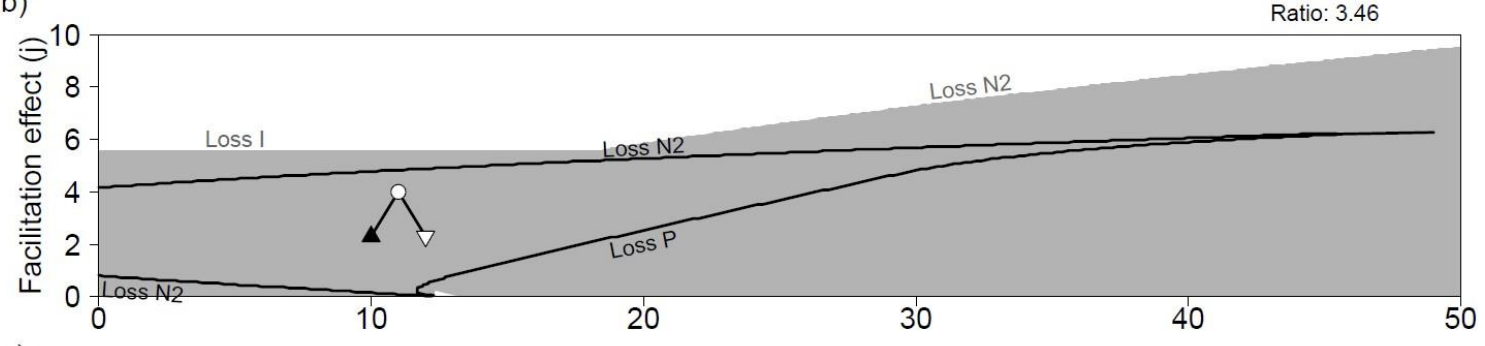

c)

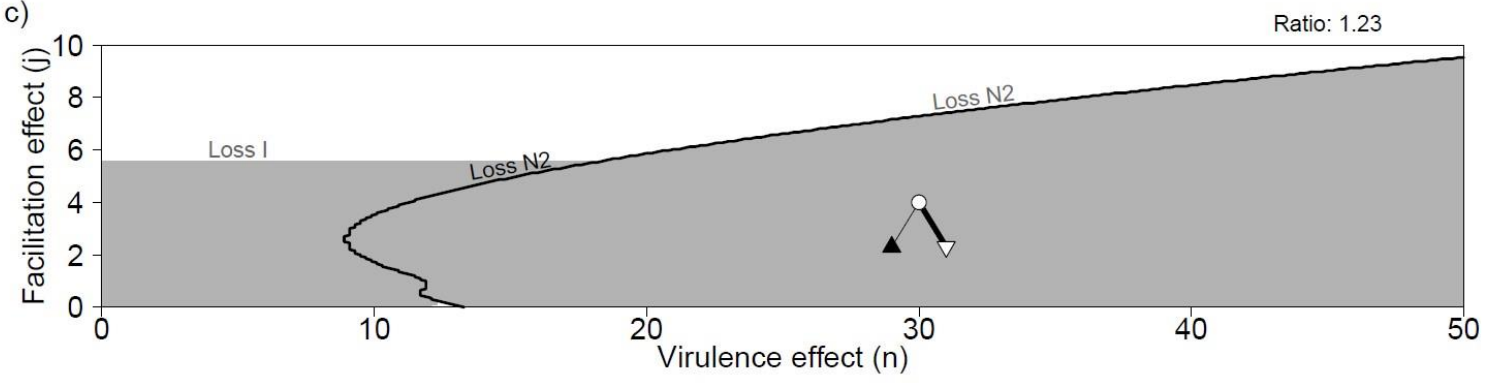

Figure B1. Coexistence of the three species, with concave trade-off, with evolution (grey area) and without evolution (inside the black delimitation). When the predator is a) specialist on the infected prey $N_{1}$, b) generalist, c) specialist on the alternative prey $N_{2}$. The species loss at the limits are written, in dark without evolution, in grey with evolution. Ratio is the ratio of coexistence surface with/without evolution. Parameters value: see Table 1 except $s=0.8$. Note that Fig. 5 shows the results with a convexe trade-off. 
561 evolution allows coexistence because it selects predator diets that are more specialized on the

562 host prey. Thereby, evolution increases apparent competition on the prey that is, without

563 evolution, competitively dominant. At high intensities, the predator might be excluded because

564 the parasite decreases the amount of prey available, especially in case of specialization on the

565 host prey (Fig. B1a). Here evolution promotes coexistence in that it favors the diets more

566 oriented toward the alternative prey. 